\title{
Dogs are more permissive than cats or guinea pigs to experimental infection with a human isolate of Bartonella rochalimae
}

\author{
Bruno B. Chomel ${ }^{1 *}$, Jennifer B. HenN ${ }^{1,2}$, Rickie W. Kasten ${ }^{1}$, Nathan C. Nieto ${ }^{3}$, \\ Janet Foley ${ }^{3}$, Sophia Papageorgiou ${ }^{3}$, Claire Allen $^{4}$, Jane E. Koehler ${ }^{5}$ \\ ${ }^{1}$ Department of Population Health and Reproduction, School of Veterinary Medicine, \\ University of California, Davis, CA 95616, USA \\ ${ }^{2}$ Napa County Public Health, 2344 Old Sonoma Road, Building F, Napa, CA 94559, USA \\ ${ }^{3}$ Department of Medicine and Epidemiology, School of Veterinary Medicine, \\ University of California, Davis, CA 95616, USA \\ ${ }^{4}$ Center for Companion Animal Health, School of Veterinary Medicine, \\ University of California, Davis, CA 95616, USA \\ ${ }^{5}$ Division of Infectious Diseases, University of California, San Francisco, 513 Parnassus Avenue, \\ Room S-380 San Francisco, CA 94143-0654, USA
}

(Received 19 August 2008; accepted 5 March 2009)

\begin{abstract}
Bartonella rochalimae was first isolated from the blood of a human who traveled to Peru and was exposed to multiple insect bites. Foxes and dogs are likely natural reservoirs for this bacterium. We report the results of experimental inoculation of two dogs, five cats and six guinea pigs with the only human isolate of this new Bartonella species. Both dogs became bacteremic for 5-7 weeks, with a peak of $10^{3}-10^{4}$ colony forming units (CFU)/mL blood. Three cats had low bacteremia levels $(<200 \mathrm{CFU} / \mathrm{mL})$ of $6-8$ weeks' duration. One cat that remained seronegative had two bacterial colonies isolated at a single culture time point. A fifth cat never became bacteremic, but seroconverted. None of the guinea pigs became bacteremic, but five seroconverted. These results suggest that dogs could be a reservoir of this strain of $B$. rochalimae, in contrast to cats and guinea pigs.
\end{abstract}

Bartonella rochalimae / dogs / cats / guinea pigs / zoonosis

\section{INTRODUCTION}

Bartonella infection was first identified in dogs in 1993, when a novel Bartonella subspecies, B. vinsonii subsp. berkhoffii (B. v. berkhoffii), was isolated from a dog with vegetative valvular endocarditis [3]. In the subsequent ten years after recognition of this organism in domestic dogs, six other species of Bartonella

\footnotetext{
* Corresponding author: bbchomel@ucdavis.edu
}

were identified in dogs, in association with various clinical manifestations [6]. To date, only two studies have been published on experimental infection of dogs with Bartonella, both of which investigated the immunological response and natural history of infection with $B . v$. berkhoffii in dogs $[18,19]$. Blood culture and polymerase chain reaction (PCR) of DNA extracted from blood or tissue suggested the establishment of chronic infection despite substantial production of $B$. v. berkhoffii-specific

This is an Open Access article distributed under the terms of the Creative Commons Attribution-Noncommercial License (http://creativecommons.org/licenses/by-nc/3.0/), which permits unrestricted use, distribution, and reproduction in any noncommercial medium, provided the original work is properly cited. 
antibodies in both studies. In one of these studies, all six $B$. v. berkhoffii inoculated dogs seroconverted by day 7 post inoculation (PI) using an indirect immunofluorescence antibody (IFA) assay, and all had elevated antibody titers ( $\geq 1: 64)$ for more than 100 days PI [19].

Bartonella rochalimae was first isolated from the blood of a human patient who presented with fever, rash, and splenomegaly after traveling to Peru where she received multiple insect bites [9]. Previously described as Bartonella clarridgeiae-like, this zoonotic Bartonella species has also been isolated from domestic dogs, gray foxes, raccoons and coyotes in California [11, 12]. Sequential infection with B. v. berkhoffii followed by B. rochalimae has been observed in a naturally infected gray fox, suggesting a lack of cross-protection between these Bartonella species [11]. $B$. rochalimae DNA (originally described as a B. clarridgeiae-like species) was also detected in one dog with endocarditis $[12,17]$. Because exposure to $B$. rochalimae likely occurred when the American woman tourist was traveling in Peru, we sought to identify which of the domestic animals usually present in traditional rural Peruvian households, i.e. dogs, cats and guinea pigs, could serve as a permissive reservoir host for $B$. rochalimae, using experimental inoculation of animals with the only human isolate available worldwide [9]. Even though the American tourist denied exposure to cats during her trip to Peru [9], and pet cats are not as common as dogs in traditional rural Peruvian households, it was nevertheless important to investigate the susceptibility of cats to this human strain, because $B$. rochalimae is most closely related genetically to $B$. clarridgeiae, a species for which cats are the natural reservoir [6]. Finally, because guinea pigs (Cavia porcellus) are commonly raised indoors, roaming free in Peruvian rural households [7, 8], we inoculated guinea pigs to investigate their role as a potential reservoir of $B$. rochalimae. Furthermore, guinea pigs are commonly infested by Pulex simulans fleas that will feed readily on humans, and a Bartonella species nearly identical to the human isolate of $B$. rochalimae [9] was identified in a Pulex flea collected on a human in Cuzco, Peru, based on the sequence of a fragment of the intergenic spacer region (ITS) [20]. Since then, B. clarridgeiae-like/ $B$. rochalimae DNA has also been detected in rodent fleas from Egypt [16], China [14] and the USA [1], and has been isolated from field mice from Slovenia [13] and a brown rat (Rattus norvegicus) from Taiwan [15], suggesting a role for rodents as reservoirs of $B$. rochalimae. We hypothesized that dogs are more likely to express higher bacteremia levels over a longer period of time than cats when inoculated with the human strain, based on previous experimental infections using a strain isolated from a wild carnivore. Because rodents could harbor B. rochalimae or B. rochalimae-like bacteria, we hypothesized that guinea-pigs are a potential reservoir for $B$. rochalimae.

Our objectives were to evaluate the susceptibility of cats and guinea-pigs to the unique human isolate of $B$. rochalimae and confirm that dogs, which have been shown to be naturally infected with $B$. rochalimae [11], represent a permissive host for infection with this human isolate. We also sought to confirm a lack of cross-protection between $B . v$. berkhoffii and $B$. rochalimae, as observed in natural infections in wild carnivores. We studied experimental infection with the only human $B$. rochalimae strain in two dogs (one dog had been inoculated previously with $B . v$. berkhoffii and the other dog had no prior exposure to Bartonella), five cats, and six guinea pigs. We determined duration of $B$. rochalimae bacteremia, antibody kinetics, and serological cross-reactivity with other Bartonella antigens for this study; these parameters have not been examined previously for $B$. rochalimae infection in domestic dogs and cats.

\section{MATERIALS AND METHODS}

\subsection{Animals}

Two mongrel male dogs (approximately 3 and 6 years old) that were seronegative and culture negative for Bartonella were obtained through the Center for Laboratory Animal Science, University of California, Davis, USA (UCD) for the study. Five 13-month-old neutered male cats that were seronegative and culture 
negative for Bartonella were obtained from the Feline Research Colony at UCD for the study. Six adult guinea pigs (three males and three females) shown to be blood culture negative and seronegative for Bartonella spp. were obtained from the Animal Science Department, UCD. All animals were raised in a flea and tick free environment, the dogs and guinea pigs having been used in other experiments prior to this study. None of these animals had been previously used in experimental infections with animal or human pathogens. The dogs, cats and guinea pigs were maintained in accordance with guidelines of the Institutional Animal Care and Use Committee (IACUC) and were examined by a veterinarian at least once to twice a week throughout the study period.

\subsection{Experimental design and bacterial culture}

For the two dogs, B. v. berkhoffii (ATCC \# 51672) or B. rochalimae (Human strain: ATCC \#BAA-1498) were plated onto $5 \%$ rabbit blood agar and incubated at $35{ }^{\circ} \mathrm{C}$ for $4-5$ days. The harvested colonies were suspended into sterile saline and a total of $0.5 \mathrm{~mL}$ was inoculated intradermally in 3 to 5 sites near the shoulder blade, as previously described [2]. Prior to inoculation with $B$. rochalimae, Dog 1 was inoculated with $2.4 \times 10^{9}$ colony forming units (CFU)/ $\mathrm{mL}$ of $B . v$. berkhoffii and blood samples collected twice a week during the first 4 weeks and then weekly for the two following months. After inoculation with $B$. rochalimae, dogs were bled at least twice a week for the first 5 weeks following inoculation and then once weekly thereafter. All blood samples (500 $\mu \mathrm{L}$ to $1 \mathrm{~mL}$ of blood per animal) were cultured on heart infusion agar containing 5\% rabbit blood and incubated in $5 \% \mathrm{CO}_{2}$ at $35{ }^{\circ} \mathrm{C}$ for up to 4 weeks $[4,5]$. After Dog 1 had been culture-negative for B. v. berkhoffii for one month, both Dog 1 and Dog 2 were inoculated with $3.5 \times 10^{8} \mathrm{CFU} / \mathrm{mL}$ of $B$. rochalimae. The two dogs were then followed using blood culture and IFA for three months. Isolates from both dogs were confirmed to be $B$. rochalimae (and not $B$. v. berkhoffii) by PCRRFLP [12] for the first positive blood culture after inoculation with $B$. rochalimae, and randomly thereafter for Dog 1.

The cats were inoculated with a total of $0.5 \mathrm{~mL}$ by the intradermal route in 3 to 4 different sites behind the shoulder blade of a $1.7 \times 10^{8} \mathrm{CFU} / \mathrm{mL}$ suspension of $B$. rochalimae (Human strain: ATCC \#BAA-1498) and clinically monitored daily for the first week. Cats were bled once a week for 15 weeks. Blood culture and serology testing were performed as previously described [23], and the first positive blood culture confirmed to be $B$. rochalimae by PCR-RFLP.

The six guinea pigs were experimentally inoculated with a total of 0.4 to $0.5 \mathrm{~mL}$ by intradermal route (in 3 to 4 different sites on the back) with a $6.1 \times 10^{8} \mathrm{CFU} / \mathrm{mL}$ suspension of $B$. rochalimae and monitored daily for the first week. A blood sample $(0.1$ to $0.2 \mathrm{~mL})$ was collected aseptically by intravenous puncture (saphenous vein) every week for 8 weeks. Blood culture and serology testing were performed as previously described [23]. Because of the low volume of whole blood collected, only 25 to $100 \mu \mathrm{L}$ of whole blood from each guinea pig was plated onto $5 \%$ rabbit blood agar.

\subsection{Serology}

For serological testing of the two dogs, only samples collected at weekly intervals were tested by IFA for antibodies against $B$. v. berkhoffii, $B$. clarridgeiae, B. rochalimae, and $B$. henselae, to examine antibody response and cross-reactivity. All IFA slides were prepared the same way by infecting Vero cells with the various strains, as previously described [11]. Cats and guinea pigs were only tested for presence of antibodies against $B$. rochalimae by IFA and a titer $\geq 1: 64$ was considered positive. For the guinea-pig samples, we used a goat anti-guinea pig conjugate at 1:100 dilution (Kirkegaard and Perry Laboratories (KPL), Gaithersburg, MD 20879, USA).

\section{RESULTS}

No physical or clinical abnormalities, except for a small focus of inflammation (redness, swelling) at the inoculation site, were observed in the dogs during the study. None of the cats or guinea pigs presented any abnormal clinical signs during the course of the study.

After inoculation with $B$. v. berkhoffii, Dog 1 developed a positive blood culture by day $17 \mathrm{PI}$ and remained culture positive through day 59 PI. The mean level of bacteremia over these 43 days was $2 \times 10^{2} \mathrm{CFU} / \mathrm{mL}$. Initial reciprocal IFA titers against $B$. v. berkhoffii were $<1: 16$, rose to $1: 64$ by day 9 PI and peaked at 1:256 within 2 weeks of the first positive blood culture (Fig. 1). The B. v. berkhoffii antibody titer was $<1: 16$ at the time of 


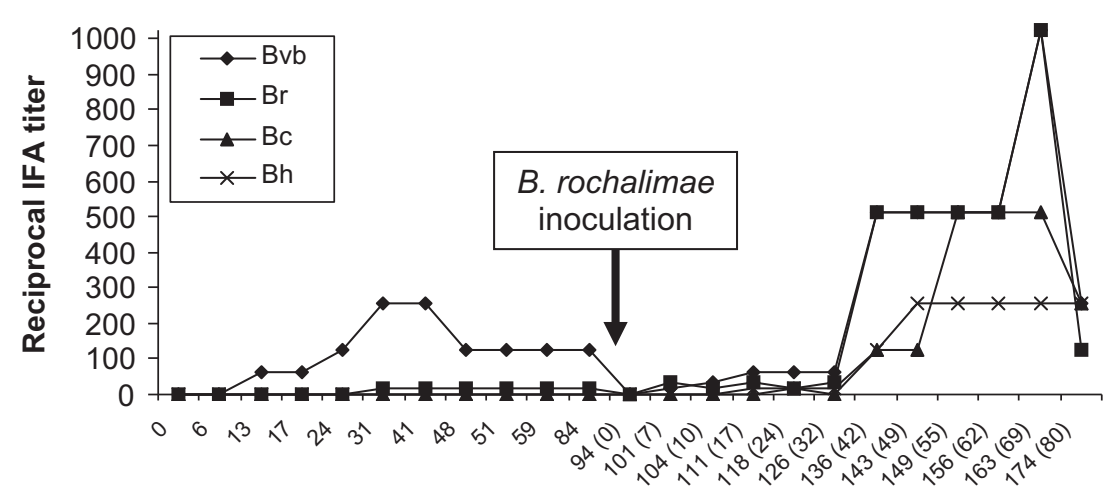

Post-inoculation day (PID)

Figure 1. IgG antibody response in Dog 1 experimentally infected with $B$. v. berkhoffii and challenged with B. rochalimae. The dog was tested by IFA for antibodies against $B$. v. berkhoffii $(\mathrm{Bvb})$, B. rochalimae $(\mathrm{Br})$, $B$. clarridgeiae $(\mathrm{Bc})$, and $B$. henselae $(\mathrm{Bh})$. The $\operatorname{dog}$ was inoculated with a strain of $B$. rochalimae on $B$. v. berkhoffii PI day 94 (day 0 in parentheses on $X$ axis).

inoculation with $B$. rochalimae, 94 days after the first inoculation with $B$. v. berkhoffii. There was no cross reactivity of anti- $B$. v. berkhoffii antibodies with antigens from other Bartonella species by IFA, and titers for B. clarridgeiae, $B$. rochalimae, and $B$. henselae remained $\leq 1: 16$ (Fig. 1). Following inoculation with $\bar{B}$. rochalimae, Dog 1 became blood culture positive for $B$. rochalimae (speciation confirmed by PCR-RFLP) at day 24 PI (118 days after inoculation with $B$. v. berkhoffii) and remained bacteremic through day 62 PI (Tab. I). A substantial increase in Bartonella IFA antibody titers was not observed until 18 days (day 42 PI) after B. rochalimae bacteremia was first observed. $B$. rochalimae and B. v. berkhoffii titers both peaked at 1:1024 on day 163 PI and demonstrated substantial cross reactivity with the other Bartonella antigens tested (Fig. 1). Dog 2, inoculated only with $B$. rochalimae, had a positive blood culture by day $10 \mathrm{PI}$ and remained culture positive through day 80 PI. Bacteremia peaked at day 17 PI $\left(5.5 \times 10^{4} \mathrm{CFU} / \mathrm{mL}\right)$ and decreased through day 42 PI. There was one negative culture on day 49 PI, followed by a second and lower increase in bacteremia with a peak of $5.1 \times 10^{2} \mathrm{CFU} / \mathrm{mL}$ on day $69 \mathrm{PI}$. Detectable levels of bacteremia ranged from $8 \mathrm{CFU} / \mathrm{mL}$ to $5.1 \times 10^{3} \mathrm{CFU} / \mathrm{mL}$ in Dog 1 and from $1 \mathrm{CFU} / \mathrm{mL}$ to $5.5 \times 10^{4} \mathrm{CFU} / \mathrm{mL}$ in Dog 2 . Mean level of bacteremia was $7.6 \times 10^{2} \mathrm{CFU} /$ $\mathrm{mL}$ for Dog 1 and $8 \times 10^{3} \mathrm{CFU} / \mathrm{mL}$ for Dog 2 . B. rochalimae IFA antibody titers $(>1: 16)$ were not observed in Dog 2 until day 24 PI, 14 days after Bartonella infection was detected by blood culture and titers increased rapidly and peaked at 1:1024 on day 32 PI. Moderate to low seroreactivity to $B$. clarridgeiae and $B$. henselae antigens was also observed in this dog, whereas B. v. berkhoffii antibody titers remained $\leq 1: 32$ (Fig. 2).

Bacteremia was detected in 4 of the 5 cats. In three cats, bacteremia was detected at week 3 PI, peaked between weeks 6 and 7, and resolved by weeks 8 to 10 . A fourth cat had a transient and low level bacteremia (2 CFU/ $\mathrm{mL}$ ) on week 12, and the fifth cat remained culture negative throughout the 15 -week study period. Overall, the level of bacteremia was low (less than $10 \mathrm{CFU} / \mathrm{mL}$ for one cat, less than $50 \mathrm{CFU} / \mathrm{mL}$ for two cats, and less than $200 \mathrm{CFU} / \mathrm{mL}$ for one cat) and at least $10-100$ times less than in the two dogs. Four of the five cats seroconverted (Tab. II). Three cats were seropositive for $B$. rochalimae 3 weeks PI, with titers ranging from 1:64 to 1:512 until the end of the study (week 15), and one cat seroconverted 
Table I. Weekly total number of colony forming units (CFU) per $\mathrm{mL}$ of blood for dogs, cats and guinea pigs experimentally inoculated with $B$. rochalimae.

\begin{tabular}{|c|c|c|c|c|c|c|c|c|}
\hline \multirow[t]{3}{*}{ Week } & \multicolumn{8}{|c|}{ Bacteremia $(\mathrm{CFU} / \mathrm{mL})$} \\
\hline & \multicolumn{2}{|c|}{ Dogs } & \multicolumn{5}{|c|}{ Cats } & \multirow{2}{*}{$\begin{array}{l}\text { Guinea pigs } \\
\quad(N=6)\end{array}$} \\
\hline & D1* & $\mathrm{D} 2$ & $\overline{\mathrm{C} 1}$ & $\mathrm{C} 2$ & $\mathrm{C} 3$ & $\mathrm{C} 4$ & $\overline{\mathrm{C} 5}$ & \\
\hline 0 & 0 & 0 & 0 & 0 & 0 & 0 & 0 & 0 \\
\hline 1 & 0 & 0 & 0 & 0 & 0 & 0 & 0 & 0 \\
\hline 2 & 0 & 392 & 0 & 0 & 0 & 0 & 0 & 0 \\
\hline 3 & 0 & $5.5 \times 10^{4}$ & 1 & 0 & 0 & 2 & 2 & 0 \\
\hline 4 & 72 & $3.3 \times 10^{4}$ & 3 & 0 & 0 & 10 & 2 & 0 \\
\hline 5 & $5 \times 10^{3}$ & $1.2 \times 10^{3}$ & 5 & 0 & 0 & 64 & 0 & 0 \\
\hline 6 & 47 & 20 & 6 & 0 & 0 & 191 & 6 & 0 \\
\hline 7 & 18 & 0 & 18 & 0 & 0 & 107 & 12 & 0 \\
\hline 8 & 57 & 1 & 4 & 0 & 0 & 3 & 46 & 0 \\
\hline 9 & 8 & 11 & 1 & 0 & 0 & 0 & 16 & ND \\
\hline 10 & 0 & 512 & 0 & 0 & 0 & 0 & 1 & ND \\
\hline 11 & 0 & 16 & 0 & 0 & 0 & 0 & 0 & ND \\
\hline 12 & 0 & 0 & 0 & 2 & 0 & 0 & 0 & ND \\
\hline 13 & 0 & 0 & 0 & 0 & 0 & 0 & 0 & ND \\
\hline 14 & 0 & 0 & 0 & 0 & 0 & 0 & 0 & ND \\
\hline 15 & 0 & 0 & 0 & 0 & 0 & 0 & 0 & ND \\
\hline
\end{tabular}

* Previously inoculated with $B$. v. berkhoffii.

$\mathrm{ND}=$ Not done.

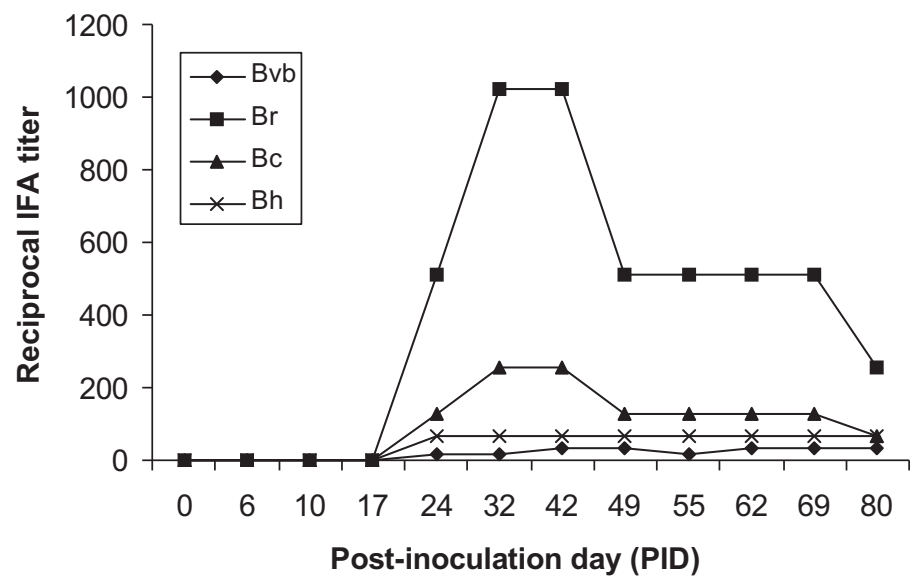

Figure 2. IgG antibody response in Dog 2 experimentally infected with B. rochalimae. This dog was tested by IFA for antibodies against $B . v$. berkhoffii $(\mathrm{Bvb}), B$. rochalimae $(\mathrm{Br})$, B. clarridgeiae $(\mathrm{Bc})$, and B. henselae (Bh).

at week 9 (titer of 1:64 until the end of the study). Interestingly, the only seronegative cat had a very low bacteremia $(2 \mathrm{CFU} / \mathrm{mL})$ at one point time (week $12 \mathrm{PI}$ ).
None of the six guinea pigs became bacteremic during the 8 weeks they were tested (Tab. I). All animals were seronegative on day 5 PI (Tab. II), three began to show B. rochalimae 
Table II. B. rochalimae antibody titers in experimentally infected cats and guinea pigs.

\begin{tabular}{|c|c|c|c|c|c|c|c|c|c|c|c|}
\hline \multirow[t]{3}{*}{ Week } & \multicolumn{11}{|c|}{ IFA antibody titers } \\
\hline & \multicolumn{5}{|c|}{ Cats } & \multicolumn{6}{|c|}{ Guinea-pigs } \\
\hline & $\mathrm{C} 1$ & $\mathrm{C} 2$ & $\mathrm{C} 3$ & $\mathrm{C} 4$ & $\mathrm{C} 5$ & GP1 & GP2 & GP3 & GP4 & GP5 & GP6 \\
\hline 0 & 0 & 0 & 0 & 0 & 0 & 0 & 0 & 0 & 0 & 0 & 0 \\
\hline 1 & 0 & 0 & 0 & 0 & 0 & 0 & 0 & 0 & 0 & 0 & 0 \\
\hline 2 & 0 & 0 & 64 & 0 & 0 & ND & ND & ND & ND & ND & ND \\
\hline 3 & 0 & 0 & 64 & 64 & 64 & 64 & 0 & 0 & 0 & 64 & 64 \\
\hline 4 & 0 & 0 & 64 & 64 & 64 & 128 & 64 & 64 & 0 & 128 & 64 \\
\hline 5 & 0 & 0 & 64 & 128 & 64 & ND & ND & ND & ND & ND & ND \\
\hline 6 & 0 & 0 & 64 & 256 & 64 & ND & ND & ND & ND & ND & ND \\
\hline 7 & 0 & 0 & 64 & 256 & 64 & ND & ND & ND & ND & ND & ND \\
\hline 8 & 0 & 0 & 64 & 256 & 64 & 128 & 128 & 128 & 0 & 128 & 64 \\
\hline 9 & 64 & 0 & 64 & 512 & 128 & ND & ND & ND & ND & ND & ND \\
\hline 10 & 64 & 0 & 64 & 256 & 128 & ND & ND & ND & ND & ND & ND \\
\hline 11 & 64 & 0 & 64 & 512 & 128 & ND & ND & ND & ND & ND & ND \\
\hline 12 & 64 & 0 & 64 & 256 & 256 & ND & ND & ND & ND & ND & ND \\
\hline 13 & 64 & 0 & 64 & 256 & 128 & ND & ND & ND & ND & ND & ND \\
\hline 14 & 64 & 0 & 64 & 256 & 256 & ND & ND & ND & ND & $\mathrm{ND}$ & ND \\
\hline 15 & 64 & 0 & 64 & 512 & 64 & ND & ND & ND & ND & ND & ND \\
\hline
\end{tabular}

$\mathrm{ND}=$ Not done

IgG seroconversion (titer $\geq 1: 64$ ) on day $19 \mathrm{PI}$. Four of the 6 animals were seropositive by day 32 PI (range 1:64 to 1:128). At the end of the study (day $53 \mathrm{PI}$ ), all but one guinea pig had seroconverted (titer range: 1:64 to 1:256).

\section{DISCUSSION}

The present study, although limited in size, demonstrates a difference in susceptibility among domestic dogs, cats, and guinea pigs to experimental infection with a human isolate of $B$. rochalimae. To date, isolates of this new Bartonella species have been cultured from mammals in the new world, including from a human who traveled to South America [9], and from gray foxes, raccoons, coyotes and domestic dogs in California $[11,12]$. In addition, $B$. rochalimae has been cultured from a red fox (Vulpes vulpes) from France ${ }^{1}$ and a strain closely related to $B$. rochalimae was recently isolated from a rat in Taiwan [15].

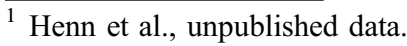

In our study, inoculation of two dogs with the human $B$. rochalimae isolate led to a successful infection, with bacteremia lasting 5 to 7 weeks and a peak bacteremia reaching $10^{3}$ to $10^{4} \mathrm{CFU} / \mathrm{mL}$. This level of bacteremia is similar to the level observed in two dogs experimentally infected with a $B$. rochalimae strain isolated from a Californian coyote ${ }^{2}$, and dogs experimentally inoculated with $B$. v. berkhoffii, another Bartonella sp. that has been isolated from canids [18, 19]. In our investigation, prior infection with $B . v$. berkhoffii in one dog was not protective against infection with $B$. rochalimae, which is consistent with observations in a naturally infected gray fox from California [11], and suggests that co-infection with these two Bartonella species could occur in domestic dogs. Similarly, in experimentally infected cats, a lack of cross-protection has been demonstrated between $B$. henselae and B. clarridgeiae [22]. Following inoculation with $B$. v. berkhoffii, Dog 1 developed only moderately elevated $(1: 256)$ IFA titers against B. v. berkhoffii but not against the other antigens

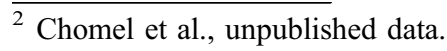


tested, and titers reverted to negative $(\leq 1: 16)$ within 35 days of the last positive blood culture. In contrast, dogs experimentally inoculated with $B . v$. berkhoffii in another study had B. v. berkhoffii antibody titers that remained elevated $(\geq 1: 64)$ for more than 100 days PI [19]. The low and rapid decline in antibody titers in Dog 1 could be a result of the failure of $B$. v. berkhoffii to establish persistent infection. In both dogs, a detectable $B$. rochalimae IgG antibody response appeared 2-3 weeks following the appearance of the first positive blood culture. Dog 1, previously exposed to B. $v$. berkhoffii, had IFA titers of $\geq 512$ against all four Bartonella antigens, whereas for Dog 2 high IFA titers were only observed for B. rochalimae antigens. Previous studies have noted a correlation between high IFA titers and seroreactivity to multiple Bartonella species $[10,21]$. These limited results suggest that infection or re-infection with multiple species of Bartonella could substantially increase the serological cross reactivity between Bartonella species. As co-infection with more than one Bartonella species has been documented in several animal species, including dogs and humans, examination of cross-reactivity in naturally-exposed animals becomes more challenging and would require immuno-absorption studies.

In contrast to observations in experimentally infected dogs, the experimentally infected cats did not show high levels of bacteremia. The peak bacteremia was above $100 \mathrm{CFU} / \mathrm{mL}$ in only one of the five cats, and was at least 10 to 100 fold lower than in experimentally infected dogs. Levels of bacteremia were also much lower than in cats experimentally infected with B. henselae or B. clarridgeiae [22-24]. Based on this small study, cats do not appear to be highly susceptible to the human isolate of $B$. rochalimae and are unlikely to constitute a natural reservoir for this human strain. There are no reports in the published literature of cats infected with $B$. rochalimae and other B. clarridgeiae-like spp. On the other hand, despite recent reports of the presence of $B$. clarridgeiae-like/B. rochalimae strains in rodents and rodent fleas, we were unable to detect infection with $B$. rochalimae in the six experimentally inoculated guinea pigs, despite a high inoculum dose $\left(10^{8} \mathrm{CFU} / \mathrm{mL}\right)$. The lack of detection could be related to the low volume of blood cultured and/or to a very low level of bacteremia. As the guinea-pigs were bled only once a week, detection of a very short term bacteremia could have been missed. Although we did not detect B. rochalimae bacteremia, the guinea pigs did develop a detectable humoral immune response to the experimental infection. Based on our data, guinea pigs do not appear to constitute a permissive reservoir for $B$. rochalimae. However, field studies should be conducted to confirm our results.

In conclusion, we sought to identify potential reservoir species from which the human strain of B. rochalimae could be acquired. We focused on animal species commonly found in the domestic environment to which the traveler could have been exposed in Peru: dogs and guinea pigs. We also included cats, because they are the natural reservoir of $B$. clarridgeiae, the Bartonella species that is closest phylogenetically to $B$. rochalimae. The dog appears to be the most permissive host of cats, dogs and guinea pigs, which is consistent with the isolation of $B$. rochalimae strains from wildlife canids. Further elucidation of reservoir hosts, including other rodent species, such as rats, as well as arthropod vectors, especially Pulex spp. fleas, for this new Bartonella species will be important in preventing infection of humans, and domestic and peri-domestic animals.

Acknowledgements. Partial funding for this project was provided through a grant from the Center for Companion Animal Health at the University of California, Davis. J.E. Koehler received funding from NIH R01 AI43703 and R01 AI52813, a California HIV/AIDS Research Program Award, and a Burroughs Wellcome Fund Clinical Scientist Award in Translational Research.

\section{REFERENCES}

[1] Abbot P., Aviles A.E., Eller L., Durden L.A., Mixed infections, cryptic diversity and vector-borne pathogens: evidence from Polygenis fleas and Bartonella species, Appl. Environ. Microbiol. (2007) 73:6045-6052.

[2] Abbott R.C., Chomel B.B., Kasten R.W., FloydHawkins K.A., Kikuchi Y., Koehler J.E., Pedersen 
N.C., Experimental and natural infection with Bartonella henselae in domestic cats, Comp. Immunol. Microbiol. Infect. Dis. (1997) 20:41-51.

[3] Breitschwerdt E.B., Kordick D.L., Malarkey D.E., Keene B., Hadfield T.L., Wilson K., Endocarditis in a dog due to infection with a novel Bartonella subspecies, J. Clin. Microbiol. (1995) 33:154-160.

[4] Chang C.C., Chomel B.B., Kasten R.W., Heller R., Kocan M., Ueno H., et al., Bartonella spp. isolated from wild and domestic ruminants in North America, Emerg. Infect. Dis. (2000) 6:306-311.

[5] Chomel B.B., MacDonald K.A., Kasten R.W., Chang C.C., Wey A.C., Foley J.E., et al., Aortic valve endocarditis in a dog due to Bartonella clarridgeiae, J. Clin. Microbiol. (2001) 39:3548-3554.

[6] Chomel B.B., Boulouis H.J., Maruyama S., Breitschwerdt E.B., Bartonella spp. in pets and effect on human health, Emerg. Infect. Dis. (2006) 12: 389-394.

[7] De la Cruz K.D., Ribbeck R., Daugschies A., Occurrence and distribution of ectoparasites in guinea pigs (Cavia spp.) in Peru, South America, Berl. Munch. Tierarztl. Wochenschr. (2003) 116:102-107 (in German).

[8] De la Cruz K.D., Whiting M.F., Genetic and phylogeographic structure of populations of Pulex simulans (Siphonaptera) in Peru inferred from two genes (CytB and CoII), Parasitol. Res. (2003) 91:55-59.

[9] Eremeeva M.E., Gerns H.L., Lydy S.L., Goo J.S., Ryan E.T., Mathew S.S., et al., Bacteremia, fever, and splenomegaly caused by a newly recognized Bartonella species, N. Engl. J. Med. (2007) 356:2381-2387.

[10] Henn J.B., Liu C.H., Kasten R.W., VanHorn B.A., Beckett L.A., Kass P.H., Chomel B.B., Seroprevalence of antibodies against Bartonella species and evaluation of risk factors and clinical signs associated with seropositivity in dogs, Am. J. Vet. Res. (2005) 66:688-694.

[11] Henn J.B., Gabriel M.W., Kasten R.W., Brown R.N., Theis J.H., Foley J.E., Chomel B.B., Gray foxes (Urocyon cinereoargenteus) as a potential reservoir of a Bartonella clarridgeiae-like bacterium and domestic dogs as sentinels for zoonotic arthropod-borne pathogens in northern California, J. Clin. Microbiol. (2007) 45:2411-2418.

[12] Henn J.B., Gabriel M.W., Kasten R.W., Brown R.N., Koehler J.E., McDonald K.A., et al., Infective endocarditis in a dog and the phylogenic relationship of the associated Bartonella rochalimae strain with isolates from dogs, gray foxes, and a human, J. Clin. Microbiol. (2009) 47:787-790.

[13] Knap N., Duh D., Birtles R., Trilar T., Petrovec M., Avsic-Zupanc T., Molecular detection of Bartonella species infecting rodents in Slovenia, FEMS Immunol. Med. Microbiol. (2007) 50:45-50.
[14] Li D.M., Liu Q.Y., Yu D.Z., Zhang J.Z., Gong Z.D., Song X.P., Phylogenetic analysis of Bartonella detected in rodent fleas in Yunnan, China, J. Wildl. Dis. (2007) 43:609-617.

[15] Lin J.W., Chen C.Y., Chen W.C., Chomel B.B., Chang C.C., Isolation of Bartonella species from rodents in Taiwan including a strain closely related to 'Bartonella rochalimae' from Rattus norvegicus, J. Med. Microbiol. (2008) 57:1496-1501.

[16] Loftis A.D., Reeves W.K., Szumlas D.E., Abbassy M.M., Helmy I.M., Moriarity J.R., Dasch G.A., Surveillance of Egyptian fleas for agents of public health significance: Anaplasma, Bartonella, Coxiella, Ehrlichia, Rickettsia, and Yersinia pestis, Am. J. Trop. Med. Hyg. (2006) 75:41-48.

[17] MacDonald K.A., Chomel B.B., Kittleson M.D., Kasten R.W., Thomas W.P., Pesavento P., A prospective study of canine infective endocarditis in Northern California (1999-2001): emergence of Bartonella as a prevalent etiologic agent, J. Vet. Intern. Med. (2004) 18:56-64.

[18] Pappalardo B.L., Brown T.T., Gebhardt D., Sontakke S., Breitschwerdt E.B., Cyclic CD8+ lymphopenia in dogs experimentally infected with Bartonella vinsonii subsp. berkhoffii, Vet. Immunol. Immunopathol. (2000) 75:43-57.

[19] Pappalardo B.L., Brown T.T., Tompkins M., Breitschwerdt E.B., Immunopathology of Bartonella vinsonii (berkhoffii) in experimentally infected dogs, Vet. Immunol. Immunopathol. (2001) 83:125-147.

[20] Parola P., Shpynov S., Montoya M., Lopez M., Houpikian P., Zeaiter Z., et al., First molecular evidence of new Bartonella spp. in fleas and a tick from Peru, Am. J. Trop. Med. Hyg. (2002) 67:135-136.

[21] Solano-Gallego L., Bradley J., Hegarty B., Sigmon B., Breitschwerdt E., Bartonella henselae IgG antibodies are prevalent in dogs from southeastern USA, Vet. Res. (2004) 35:585-595.

[22] Yamamoto K., Chomel B.B., Kasten R.W., Chang C.C., Tseggai T., Decker P.R., et al., Homologous protection but lack of heterologous-protection by various species and types of Bartonella in specific pathogen-free cats, Vet. Immunol. Immunopathol. (1998) 65:191-204.

[23] Yamamoto K., Chomel B.B., Kasten R.W., Hew C.M., Weber D.K., Lee W.I., Experimental infection of specific pathogen free (SPF) cats with two different strains of Bartonella henselae type I: a comparative study, Vet. Res. (2002) 33:669-684.

[24] Yamamoto K., Chomel B.B., Kasten R.W., Hew C.M., Weber D.K., Lee W.I., et al., Infection and re-infection of domestic cats with various Bartonella species or types: $B$. henselae type $\mathrm{I}$ is protective against heterologous challenge with B. henselae type II, Vet. Microbiol. (2003) 92:73-86. 